\title{
Comparison of the WHO/ISUP Classification and Cytokeratin 20 Expression in Predicting the Behavior of Low-Grade Papillary Urothelial Tumors
}

\author{
A. Alsheikh, M.D., Z. Mohamedali, M.D., Ph.D., E. Jones, M.D., J. Masterson, M.D., C.B. Gilks, M.D. \\ Departments of Pathology (AA, EJ, CBG) and Urology (ZM, JM), Vancouver Hospital and University of \\ British Columbia, Vancouver, British Columbia, Canada
}

It has not been possible to identify those low-grade papillary transitional cell bladder tumors that will recur based on conventional histopathologic assessment. Both the new World Health Organization/ International Society of Urologic Pathology (WHO/ ISUP) classification of transitional cell papillary neoplasms and the pattern of tumor cytokeratin 20 (CK20) immunostaining have been suggested as means of improving prognostication in low-grade transitional cell tumors. Forty-nine low-grade, noninvasive papillary transitional cell tumors were identified for the period between 1984 and 1993. The recently described WHO/ISUP classification was applied, and the tumors were classified histologically as papilloma, papillary neoplasm of low malignant potential (LMP) or low-grade papillary carcinoma. After CK20 immunostaining, the expression pattern in the tumor was classified as normal (superficial) or abnormal. Of 49 tumors, 20 were classified as papillary neoplasms of LMP and five of these patients (25\%) experienced a recurrence. Of 29 tumors classified as low-grade papillary carcinoma, $14(48.2 \%)$ recurred. In 46 of 49 cases, the CK20 immunostaining could be evaluated. Sixteen tumors showed normal (superficial) pattern of CK20 expression, and four (25\%) of these patients experienced a recurrence. In contrast, of 30 patients with abnormal CK20 staining of their tumors, 15 $(50 \%)$ patients had one or more recurrences. In this study, papillary neoplasms of LMP (as per the WHO/ ISUP classification system) had a lower recurrence rate than low-grade papillary transitional cell carcinoma. Similarly low-grade urothelial tumors showing a normal CK20 expression pattern recurred less frequently than tumors with an abnormal pattern

Copyright (C) 2001 by The United States and Canadian Academy of Pathology, Inc.

VOL. 14, NO. 4, P. 267, 2001 Printed in the U.S.A.

Date of acceptance: August 28, 2000.

Address reprint requests to: Dr. B. Gilks, Department of Pathology, Room

G227, UBC Hospital, 2211 Wesbrook Mall, Vancouver, B.C. V6T-2B5,

Canada; fax: 604-822-7575; e-mail: bgilks@vanhosp.bc.ca. of CK20 staining. Neither of these differences was statistically significant, and recurrences were observed in $20 \%$ of patients whose tumors were both classified as papillary neoplasms of LMP and showed normal CK20 immunostaining; thus they do not allow a change in our current management of patients with low-grade papillary urothelial tumors, with close follow-up for all patients.

KEY WORDS: Papillary urothelial tumors, Cytokeratin 20.

Mod Pathol 2001;14(4):267-272

The World Health Organization (WHO) and the International Society of Urologic Pathology (ISUP) have recently published a consensus classification for urothelial papillary tumors (1). This new classification system recognizes papilloma, papillary neoplasm of low malignant potential (LMP), and low-grade papillary transitional cell carcinoma at the low-grade end of the transitional cell neoplasia spectrum. A recent study has shown that this new classification of low-grade bladder tumors can provide prognostic information (2).

Cytokeratins are a family of intermediatefilament polypeptides present in epithelial cells (3). Cytokeratin 20 (CK20) is a recently described cytokeratin isotype, which shows a limited pattern of expression in normal tissues, being restricted to the gastric and intestinal epithelium, urothelium, and Merkel cells (4). It has been suggested that the pattern of CK20 staining is a useful adjunct to morphology in the diagnosis of urothelial dysplasia (5). It has also been suggested that CK20 expression can predict malignant potential in low-grade transitional cell tumors (6), and therefore CK20 can be useful in defining treatment strategies for patients with these tumors (7).

In this study, we have tested the ability of the new WHO/ISUP classification and CK20 expression, either separately or together, to predict for recurrence potential of low-grade papillary urothelial tu- 
mors. If a subset of patients with low-grade papillary transitional cell tumors who have a negligible chance of recurrence could be identified, based on pathologic examination of the resected tumors, these patients could be spared the regular cystoscopic examination that is the cornerstone of our current management of these patients.

\section{MATERIALS AND METHODS}

In this retrospective study, we searched the medical records of a single urologist (J.M.) at Vancouver Hospital-UBC Hospital Site, for the period 1984 to
1993, to identify all low-grade papillary urothelial tumors. All high-grade tumors or tumors with invasion were excluded. A total of 49 patients with lowgrade papillary transitional cell tumors were identified. All slides were reviewed by two pathologists (A.A. and C.B.G.) and the new WHO/ISUP classification was applied, with the tumors classified as papilloma, papillary neoplasm of LMP, or lowgrade papillary transitional cell carcinoma (Fig. 1). All tumors were stained for CK20 using the streptavidin-biotin method and the monoclonal antibody Ks20.8 (Dako Diagnostics Canada Inc.), which was applied to formalin-fixed, paraffin-
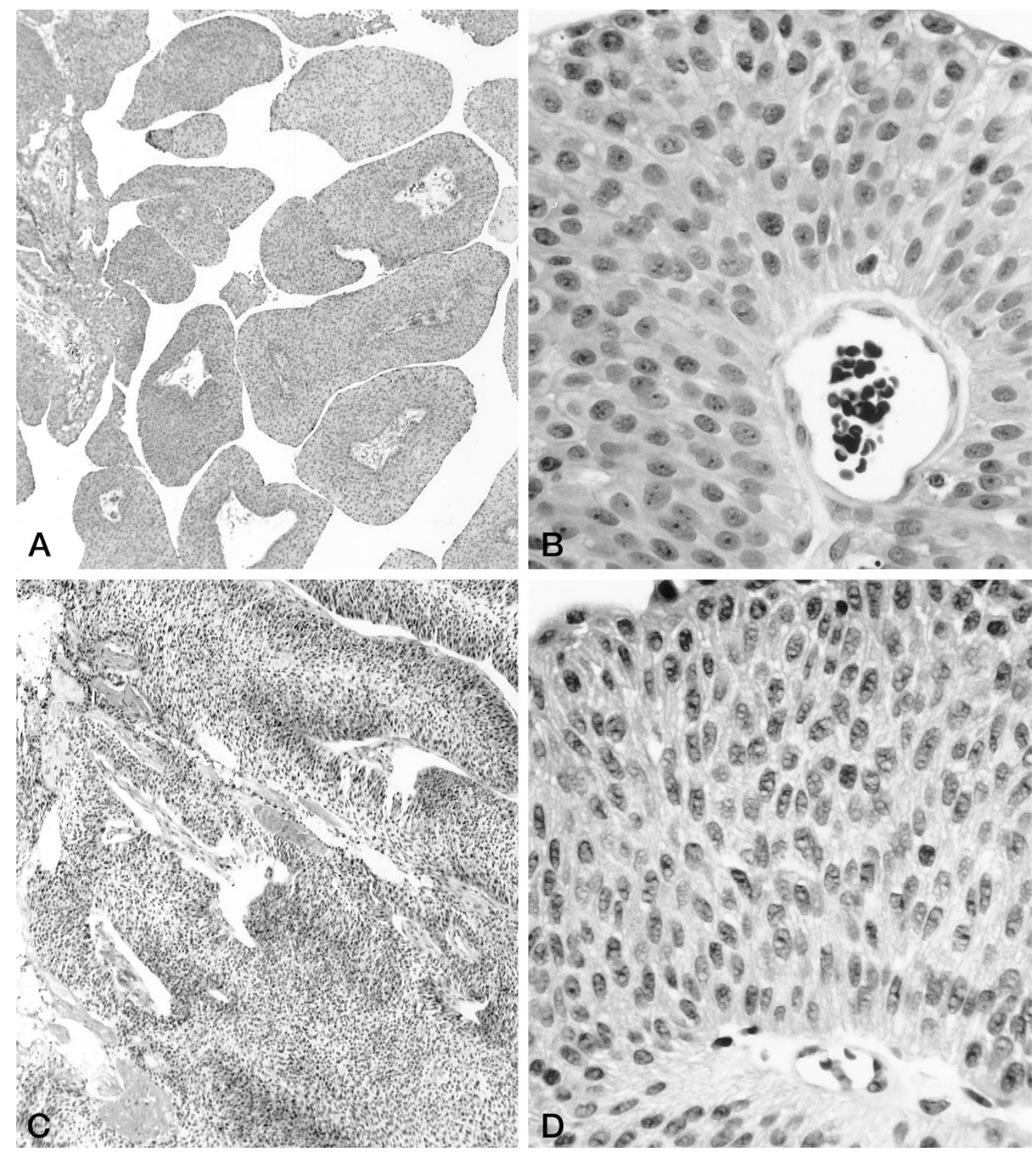

FIGURE 1. Low- and high-power photomicrographs of typical examples of papillary tumor of low malignant potential (A and B) and low-grade transitional cell carcinoma (C and D). Note the increased architectural complexity, cellular stratification, and cytologic atypia in the low-grade transitional cell carcinoma compared with the papillary tumor of low malignant potential. 
embedded tissue sections. The immunostained slides were assessed by the same pathologists, and the cases were divided according to the pattern of CK20 expression (Fig. 2). If the expression was restricted to superficial (umbrella) cells, it was classified as normal expression. Other patterns of CK 20 immunostaining, i.e., diffuse full-thickness positiv-

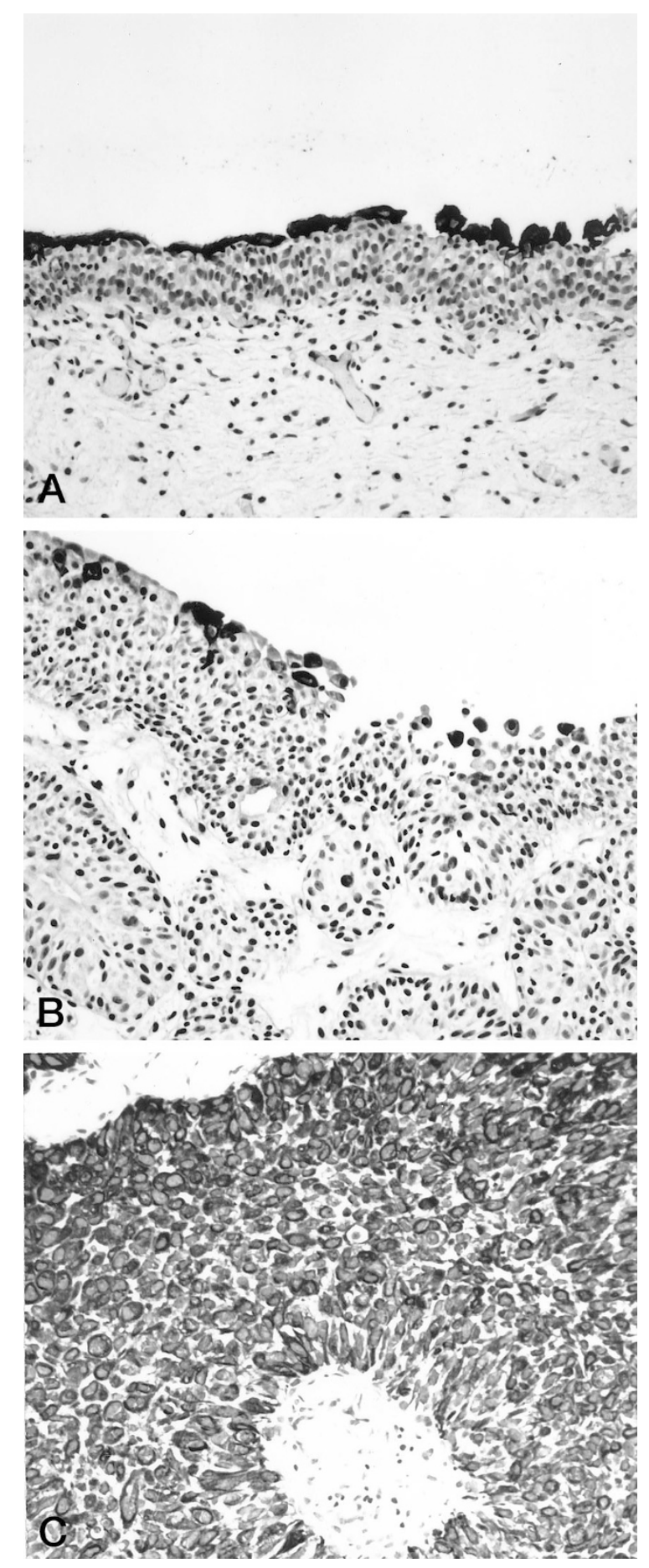

FIGURE 2. CK20 expression in normal urothelium and low-grade papillary urothelial tumors. A, Normal urothelium with superficial CK20 expression. B, Papillary urothelial tumor with normal CK20 expression, confined to the superficial urothelial cell layer. C, Papillary urothelial tumor showing abnormal, full-thickness, CK20 expression. ity or negativity for CK20 expression, were considered abnormal. Statistical analyses were done using Fisher's exact test.

The follow-up protocol after a cystoscopically directed biopsy positive for low-grade papillary transitional cell tumor was cystoscopy every 3 months for the first year, every 6 months for the second year, and then annually. All patient's files were reviewed for clinical follow-up through 1999, and slides from all recurrences were reviewed.

\section{RESULTS}

The patients range in age from 40 to 94 years (mean 76 years). Thirty-three patients are male and 16 are female. Of the 49 patients, 28 are cigarette smokers. Initial treatment consisted of transurethral resection of tumor without adjuvant therapy, e.g., BCG or intravesical chemotherapy. Of 49 tumors, 20 were classified as papillary neoplasms of LMP, and 29 were classified as low-grade papillary carcinoma (Fig. 1). No cases of papilloma were identified. Clinical follow up showed that 5 of 20 (25\%) tumors classified as papillary neoplasms of LMP had recurred (Table 1), with no evidence of progression to either low-grade or high-grade papillary carcinoma, or myoinvasion, in the recurrences. Fourteen of 29 (48.2\%) tumors classified as low-grade papillary carcinoma recurred (Table 1). In two of these cases there was progression to highgrade carcinoma, with invasion of muscularis propria in both cases, and two patients developed high-grade transitional cell carcinoma in situ. There was no evidence of progression to high-grade carcinoma in the remaining 10 cases.

Of the recurrent papillary neoplasms of LMP, one tumor recurred three times during the study period; the other patients experienced a single recurrence only, with the tumor-free period before recurrence ranging from 4 months to 7 years (mean 29 months, median 17 months). Of the cases of recurrent lowgrade papillary carcinoma, seven tumors recurred only once, one tumor recurred twice, three tumors recurred three times and three tumors recurred four times, with the tumor-free interval ranging from 5 weeks to 8 years (mean 22 months, median 15 months).

CK20 immunostaining could be evaluated in 46 out of 49 cases only. Sixteen tumors showed normal

TABLE 1. Papillary Neoplasm of LMP versus Low-Grade Papillary TCC

\begin{tabular}{lcc}
\hline & No Recurrence & Recurrence \\
\hline Papillary neoplasms of LMP & $15(75 \%)$ & $5(25 \%)$ \\
Low-grade papillary TCC & $15(51.8 \%)$ & $14(48.2 \%)$ \\
& \multicolumn{2}{c}{$0.25>P>0.10$} \\
\hline
\end{tabular}

LMP, low malignant potential; TCC, low-grade transitional cell carcinoma. 
(superficial) staining pattern of CK20 expression, and 30 tumors showed abnormal CK20 expression, including three cases with CK 20 negativity of the full thickness of the urothelium (Fig. 2). Four of 16 (25\%) tumors with normal CK20 expression had recurred (Table 2). One patient developed highgrade transitional cell carcinoma in situ, but the remaining recurrences showed no evidence of progression to higher grade tumor. In contrast, of 30 tumors with abnormal CK20 expression, 15 (50\%) had recurred (Table 2). In two of these cases there was progression to high-grade carcinoma; in both cases there was invasion of muscularis propria. A single patient developed high-grade transitional cell carcinoma in situ. There was no evidence of progression to high-grade tumor in the remaining cases. All three tumors with full thickness CK20 negativity recurred.

All tumors with normal CK20 staining pattern that recurred, have recurred only once, except for a single case with three recurrences, and the interval to recurrence ranged from 11 months to 8 years (mean 42 months). Among the tumors with abnormal CK20 staining pattern that recurred, eight tumors have recurred once only, one tumor recurred twice, three tumors recurred three times and three tumors recurred four times, with recurrence intervals of between 5 weeks and 3 years (mean 19 months, median 15 months).

The relationship between the WHO/ISUP classification and CK20 staining is shown in Table 3. Most tumors of LMP showed normal CK20 immunoreactivity (10 of 17 cases), whereas most lowgrade transitional cell carcinomas showed abnormal CK20 immunoreactivity (23 of 29 cases). Of 10 tumors that were classified as tumors of LMP and also showed normal CK20 staining pattern, two have recurred (20\%).

\section{DISCUSSION}

Low-grade papillary urothelial tumors of the urinary bladder are characterized by a high rate of recurrence and low potential for invasion $(8,9)$. Patients with low-grade urothelial tumors treated by transurethral resection typically then are monitored by regular cystoscopy, which is expensive and has potential morbidity and mortality, related primarily to the risk of anesthesia. A less invasive and

TABLE 2. Normal versus Abnormal CK20 Staining

\begin{tabular}{lcr}
\hline & No Recurrence & Recurrence \\
\hline Normal pattern of CK20 & $12(75 \%)$ & $4(25 \%)$ \\
Abnormal pattern of CK20 & $15(50 \%)$ & $15(50 \%)$ \\
& $0.25>P>0.10$ \\
\hline
\end{tabular}

CK, cytokeratin.
TABLE 3. WHO/ISUP Classification and CK20 Staining

\begin{tabular}{lcc}
\hline & No Recurrence & Recurrence \\
\hline LMP/CK20 normal & 8 & 2 \\
LMP/CK20 abnormal & 4 & 3 \\
TCC/CK20 normal & 4 & 2 \\
TCC/CK20 abnormal & 11 & 12 \\
\hline
\end{tabular}

LMP, low malignant potential; TCC, low-grade transitional cell carcinoma; CK, cytokeratin.

expensive alternative to cystoscopy is desirable and a variety of alternatives have been investigated.

Urine cytology has been used for follow-up in patients with bladder carcinoma (10). Cytology is effective in monitoring recurrence of high-grade urothelial tumors, but is ineffective in low-grade papillary urothelial tumors because the tumor cells lack many of the cytologic features of malignancy $(10,11)$.

The ability of flow cytometry of exfoliated tumor cells to detect recurrent urothelial tumors has also been evaluated. Flow cytometry and image analysis can detect DNA aneuploidy, which is characteristic of high-grade tumors but not low-grade tumors (12). Thus, for patients with low-grade papillary transitional cell tumors, analysis of exfoliated cells for DNA content is a very insensitive assay and cannot replace regular cystoscopic examination (13).

Immunohistochemical study of exfoliated urothelial cells for overexpression of Lewis $\mathrm{X}$ antigen can increase the sensitivity of urine cytologic examination (14). The main drawback has been lack of specificity, with expression of Lewis $\mathrm{X}$ antigen frequently observed in reactive urothelium (15). Detection of p53 mutations, which are frequently manifest as increased intracytoplasmic protein detected by immunohistochemistry, has similarly been suggested as a marker of transitional cell neoplasia (16), but use of immunohistochemical detection suffers from both lack of sensitivity and specificity (17). Direct mutation analysis of the p53 gene is too expensive for use as a routine diagnostic test with available technologies.

Another strategy for monitoring patients for recurrent bladder tumors is to assay urine for noncellular elements associated with transitional cell neoplasia. Examples include bladder tumor antigen, nuclear matrix proteins, fibrin/fibrinogen degradation products, telomerase and hyaluronic acid/ hyaluronidase (reviewed in ref. 18). Although there have been promising results with these assays, particularly bladder tumor antigen and telomerase (19-21), they are preliminary and larger prospective studies demonstrating their efficacy are needed. At the present time their role in follow-up of patients with low-grade transitional cell tumors is not well delineated.

Studies of biopsy specimens from low-grade papillary tumors have been undertaken in an attempt to identify features predictive of recurrence. Helpap 
and Kollerman found an increased likelihood of recurrence in tumors with a high proliferative index, assessed MIB-1 immunostaining (22). Tsujihashi et al. showed that immunostaining for bromodeoxyuridine incorporation and Ki-67 also correlated with grade although the ability of these proliferation markers to predict outcome was not tested directly (23). Although markers of tumor cell proliferation hold promise as prognostic indicators for patients with low-grade papillary transitional cell tumors, further studies are needed.

The WHO/IUSP have recently published a consensus classification for urothelial papillary tumors (1). In this new classification, they have divided the low-grade urothelial tumors into urothelial neoplasms of LMP and low-grade urothelial carcinoma, both of which were regarded as transitional cell carcinoma, grade 1, in the old WHO classification. Subsequently, Holmang et al. showed that papillary neoplasms of LMP have a lower rate of recurrence than low-grade papillary carcinoma (2), a finding identical to the present study. This difference does not reach statistical significance in the present study, which may reflect the small size of this study, with only 49 cases.

Harnden et al. have suggested that assessment of CK20 expression can be used as a predictor for recurrence rate and malignant potential in lowgrade urothelial tumors, concluding that tumors with abnormal CK20 expression were more likely to recur $(6,7)$.

In this study, we showed that papillary neoplasms of LMP had a lower recurrence rate than low-grade papillary transitional carcinoma, and that low-grade urothelial tumors with normal CK20 expression pattern recurred less frequently than tumors with abnormal pattern of CK20 staining. As well, patients with either low-grade papillary carcinomas or abnormal CK20 immunostaining patterns experienced more recurrences and more frequently had invasion in the recurrent tumors, compared with patients with papillary tumors of LMP or normal CK20 immunostaining. However, even tumors that were both classified as papillary tumors of LMP and showed normal CK20 expression recurred in $20 \%$ of cases. Thus it did not prove possible to identify a subset of low-grade papillary urothelial tumors with a sufficiently low likelihood of recurrence that it would allow a change in our current management of these patients with long-term close clinical follow-up, including regular cystoscopic examination.

A limitation of this study is that the end point used, recurrence of tumor, is less important clinically than recurrence of invasive tumor or death due to tumor. The ideal prognostic indicator will identify those patients at risk of developing invasion in recurrent tumor so that these patients may be followed more closely or, conceivably, offered active intervention to eradicate the malignant clone that leads to metachronous recurrences (24). As invasive recurrences are uncommon, ultimately occurring in approximately $15 \%$ of patients with lowgrade transitional cell tumors (9), a much larger number of patients will be needed to study prognostic indicators in patients with low-grade transitional cell tumors that are predictive of progression to invasive tumor. In this small series there were only two recurrences with invasion of the muscularis propria (pathologic stage T2) and both the new WHO/ISUP classification (i.e., low-grade urothelial carcinoma rather than papillary tumor of LMP), and abnormal CK20 staining identified these patients.

\section{REFERENCES}

1. Epstein J, Amin M, Reuter V, Mostofi FK and the Bladder Consensus Conference Committee. The World Health Organization/International Society of Urological Pathology Consensus Classification of Urothelial (Transitional cell) Neoplasms of the Urinary Bladder. Am J Surg Pathol 1998;22: 1435-48.

2. Holmang S, Hedelin H, Anderstrom C, Holmberg E, Busch C, Johansson SL. Recurrence and progression in low grade papillary urothelial tumors. J Urol 1999;162:702-7.

3. Moll R. Molecular diversity of cytokeratin: significance for cell and tumor differentiation. Acta Histochem Suppl 1991; 4:117-27

4. Moll R, Lowe A, Laufer J, Franke WW. Cytokeratin 20 in human carcinomas: a new histodiagnostic marker detected by monoclonal antibodies. Am J Pathol 1992;140:427-47.

5. Harnden P, Eardley I, Joyce AD, Southgate J. Cytokeratin 20 as an objective marker of urothelial dysplasia. Br J Urol 1996;78:870-5.

6. Harnden P, Mahmood N, Southgate J. Expression of cytokeratin 20 redefines urothelial papillomas of the bladder. Lancet 1999;353:974-7.

7. Harnden P, Allam A, Joyce AD, Patel A, Selby P, Southgate J. Cytokeratin 20 expression by non-invasive transitional cell carcinomas: potential for distinguishing recurrent from nonrecurrent disease. Histopathology 1995;27:169-74.

8. Koss LG. Tumors of the urinary bladder. In: Atlas of Tumor Pathology, Second Series, Fascicle 11. Washington, DC: Armed Forces Institute of Pathology; 1975 (Suppl. 1985).

9. Lee R, Droller MJ. The natural history of bladder cancer: implications for therapy. Urol Clin North Am 2000;27:1-13.

10. Murphy WM. Current status of urinary cytology in the evaluation of bladder neoplasms. Hum Pathol 1990; 21:886-96.

11. Koss LG, Deitch D, Ramanathan R, Sherman AB. Diagnostic value of cytology of voided urine. Acta Cytol 1985;29:810-6.

12. Koss LG, Wersto RP, Simmons DA, Deitch D, Herz F, Freed SZ. Predictive value of DNA measurements in bladder washings: comparison of flow cytometry, image cytophotometry, and cytology in patients with a past history of urothelial tumors. Cancer 1989;64:916-24.

13. Gourlay W, Chan V, Gilks CB. Screening for urothelial malignancies by cytology and flow cytometry in a community urologic practice: a prospective study. Mod Pathol 1995;8: 394-7.

14. Pode D, Golijanin D, Sherman Y, Lebensart P, Shapiro A. Immunostaining of Lewis $\mathrm{X}$ in cells from voided urine, cytopathology and ultrasound for non-invasive detection of bladder tumors. J Urol 1998;159:389-92. 
15. Loy TS, Alexander CJ, Calaluce RD. Lewis X antigen immunostaining in the diagnosis of transitional cell carcinoma. Mod Pathol 1995;8:587-90.

16. Sidransky D, Von Eshenbach A, Tsai YC, Jones P, Summerhayes I, Marshall F, et al. Identification of p53 gene mutations in bladder cancers and urine samples. Science 1991; 252:706-9.

17. Righi E, Rossi G, Ferrari G, Dotti A, De Gaetani C, Ferrari P, et al. Does p53 immunostaining improve diagnostic accuracy in urine cytology? Diagn Cytopathol 1997;17:436-9.

18. Brown FM. Urine cytology: is it still the gold standard for screening? Urol Clin North Am 2000;27:25-37.

19. Malkowicz SB. The application of human complement factor H-related protein (BTA TRAK) in monitoring patients with bladder cancer. Urol Clin North Am 2000;27:63-73.
20. Grossman HB. New methods for detection of bladder cancer. Semin Urol Oncol 1998;16:17-22.

21. Ramakumar S, Bhuiyan J, Besse JA, Roberts SG, Wollan PC, Blute ML, et al. Comparison of screening methods in the detection of bladder cancer. J Urol 1999;161:388-94.

22. Helpap B, Kollermann J. Proliferative pattern of exophytic and superficially invasive and noninvasive low-grade urothelial carcinomas. Hum Pathol 1999;30:145-50.

23. Tsujihashi H, Nakanishi A, Matsuda H, Uejima S, Kurita T. Cell proliferation of human tumors determined by BRDURD and KI-67 immunostaining. J Urol 1991;145:846-9.

24. Li M, Cannizarro LA. Identical clonal origin of synchronous and metachronous low-grade, non-invasive papillary transitional cell carcinomas of the urinary tract. Hum Pathol 1999; 30:1197-200.

\section{Book Review}

\section{Percy DH, Barthold SW: Pathology of Labora- tory Rodents and Rabbits, 315 pp, Ames IA, lowa State University Press, 2001 (\$99.95).}

The use of rodents in laboratory research is expanding, particularly relative to the production of transgenic models. Rodent models are becoming more refined, and with that refinement there is increasing potential for impact by infectious disease on many of these models. Given the worldwide mobility of people, animals, and biomaterials, diseases from virtually any part of the world could break in US colonies. The evaluation of rodent models and particularly transgenic models requires sound reference material on the normal anatomy and on the pathologic lesions associated with common diseases in these species. Pathology of Laboratory Rodents and Rabbits provides an organized and detailed review of the important natural diseases of mice, rats, hamsters, gerbils, guinea pigs, and rabbits. It is written by two individuals who are well-known and respected in the field of rodent pathology. Each chapter provides a foundation in normal gross and microscopic features and variations that are unique to each spe- cies. All chapters are organized in a similar format, covering the common viral, bacterial, fungal, or parasitic diseases, as well as other degenerative and neoplastic lesions. The coverage of neoplastic disease is limited to only the most common tumors. High-quality photographs accompany the descriptions of the most important diseases. The significance of mouse or rat strain on disease expression also is covered well in the text. Nomenclature and taxonomy of the disease-causing organisms are current. Reference lists are clustered by disease entity with classical and recent publications. Information is so current that a recent (year 2000) outbreak of rabbit calicivirus is included. The text is a necessity for any veterinary pathologist or veterinary trainee working in rodent research, but it also would be useful for investigators who may be trying to decipher the significance of lesions in genetically modified or immunodeficient laboratory animals.

\section{David M. Pinson \\ University of Kansas Medical Center \\ Kansas City, Kansas}

